$\underline{\text { Preprint typeset in JHEP style. - PAPER VERSION }}$

\title{
Two-loop enhancement factor for $1 / Q$ corrections to event shapes in deep inelastic scattering
}

\author{
Mrinal Dasgupta and Bryan R. Webber* \\ Cavendish Laboratory, University of Cambridge, \\ Madingley Road, Cambridge CB3 OHE, U.K. \\ E-mail: dasgupta@hep.phy.cam.ac.uk, etc.
}

\begin{abstract}
We compute the two-loop enhancement factors for our earlier one-loop calculations of leading $(1 / Q)$ power corrections to the mean values of some event shape variables in deep inelastic lepton scattering. The enhancement is found to be equal to the universal "Milan factor" for those shape variables considered, provided the one-loop calculation is performed in a particular way. As a result, the phenomenology of power corrections to DIS event shapes remains largely unaffected.
\end{abstract}

KEYwORDS: QCD, NLO computations, jets, deep inelastic scattering.

\footnotetext{
${ }^{*}$ Research supported in part by the U.K. Particle Physics and Astronomy Research Council and by the EC Programme "Training and Mobility of Researchers", Network "Hadronic Physics with High Energy Electromagnetic Probes", contract ERB FMRX-CT96-0008.
} 


\section{Contents}

1. Introduction 1

2. Kinematics 2

3. DIS event shape variables 4

3.1 Current jet thrust 4

3.2 Current jet mass 5

$3.3 C$-parameter $\quad 5$

4. Soft two-loop contributions 6

5. Dispersive calculation of power corrections $\quad 8$

6. Power corrections to DIS event shapes 10

6.1 Current jet mass 11

6.2 Current jet thrust 12

$6.3 C$-parameter 13

7. Conclusions 13

\section{Introduction}

In a recent paper [1] we presented a theoretical study of the leading power-suppressed corrections to the mean values of various event-shape measures in deep inelastic scattering (DIS). Our investigation was based on an analysis of one-loop Feynman graphs containing a massive gluon, which probes the sensitivity of different observables to long-distance physics $[2,3,4]$ and is closely related to the analysis of infrared renormalons $[5,6,7,8]$. We found that, as in $e^{+} e^{-}$annihilation, the leading corrections to DIS event shapes are normally of order $1 / Q$. This prediction has since been verified experimentally [9].

By making the further assumption of universal low-energy behaviour of the strong coupling, we also estimated the magnitudes of $1 / Q$ corrections to event shapes in DIS relative to those observed in $e^{+} e^{-}$annihilation $[10,11]$. Here the one-loop massive-gluon analysis suffers from some ambiguities and deficiencies. The ambiguities arise from the sensitivity of some event shapes to the way in which the gluon 
mass is included in the definition of the shape variable, the phase space and the matrix elements. This problem is related to the inadequacy of the massive-gluon approach pointed out by Nason and Seymour [12]: unlike the total $e^{+} e^{-}$annihilation cross section, for example, event shapes are sensitive to the precise way in which a virtual gluon fragments into observable particles. In other words, they are not sufficiently inclusive to be expressible in terms of the distribution of an effectively massive gluon.

In a two-loop analysis of this problem in $e^{+} e^{-}$event shapes, however, the Milan group [13] has found that, for a wide class of shape variables, the effects of non-inclusiveness amount simply to an enhancement of the "naive massive-gluon" estimate of the $1 / Q$ correction by a universal "Milan factor", with numerical value $\mathcal{M} \simeq 1.49$. The two-loop analysis also clarified the way in which the gluon mass should be included at the "naive" level, in order that universality should be manifest.

In the present paper we perform the same type of two-loop analysis for event shape variables in DIS. We find a universal enhancement of the $1 / Q$ corrections to the current jet thrust, mass and $C$-parameter, with the same enhancement factor $\mathcal{M}$, provided the "naive massive-gluon" estimate is computed in a particular way. This resolves the ambiguities we encountered in the one-loop calculation of power corrections to certain DIS shape variables.

In the case of the current jet broadening variable, which we also studied at oneloop level in Ref. [1], there are additional complications arising from recoil contributions [14], and therefore we postpone the two-loop analysis to a future publication.

The layout of the present paper is as follows. In Sect. 2 we define convenient kinematic variables for the study of multi-parton emission in DIS. These variables are used in Sect. 3 to express the various event shape variables that we investigate. We shall argue that the dominant two-loop contributions to the leading power correction come from the kinematic region in which the emitted gluons are soft. The contributions from this region are described in Sect. 4. Sect. 5 explains the 'dispersive' method we use to calculate the power corrections. The actual calculations are then performed in Sect. 6, and finally the results are summarized and discussed in Sect. 7.

\section{Kinematics}

It is convenient to write the momenta $k_{i}$ of radiated partons (gluons and/or quarkantiquark pairs) in terms of Sudakov (light-cone) variables as (Fig. 1)

$$
k_{i}=\alpha_{i} P+\beta_{i} P^{\prime}+k_{t i}, \quad \alpha_{i} \beta_{i}=\vec{k}_{t i}^{2} / Q^{2}
$$




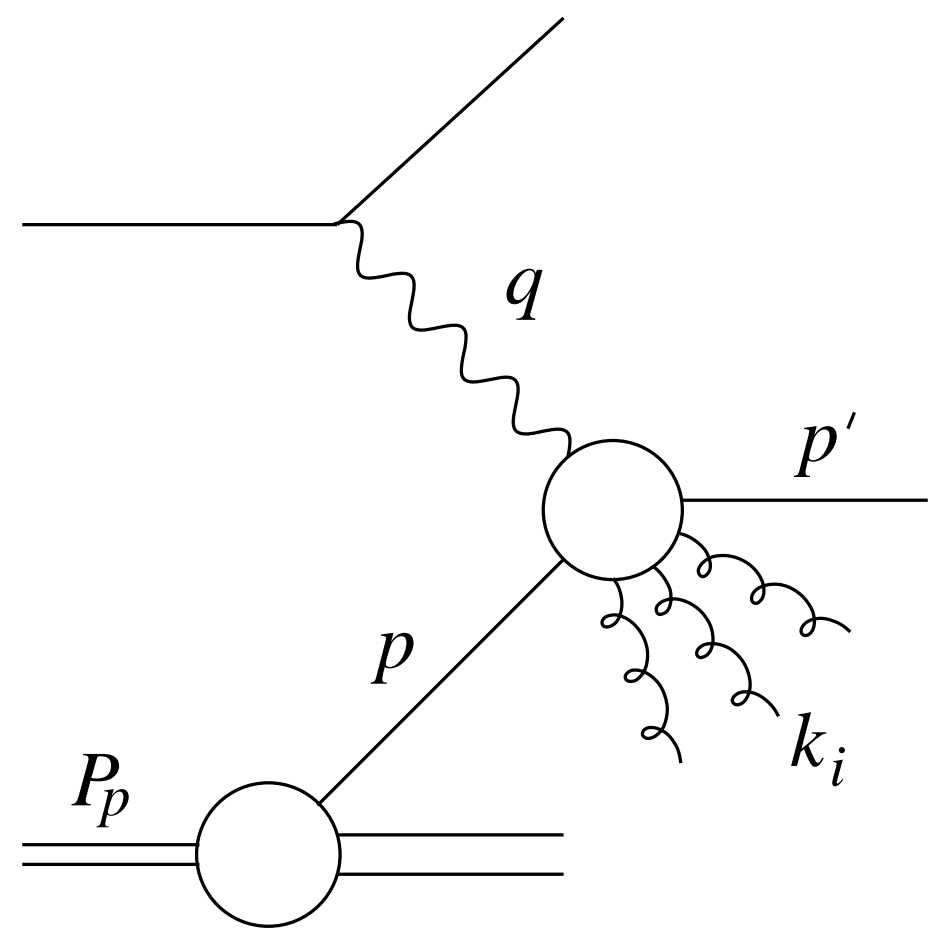

Figure 1: Deep inelastic scattering with multi-parton emission.

where $P$ and $P^{\prime}$ are light-like vectors along the incoming parton and current directions, respectively, in the Breit frame of reference:

$$
P=x P_{p}, P^{\prime}=x P_{p}+q, 2\left(P \cdot P^{\prime}\right)=-q^{2} \equiv Q^{2}
$$

where $P_{p}$ is the incoming proton momentum and $x=Q^{2} / 2\left(P_{p} \cdot q\right)$ is the Bjorken variable. Thus in the Breit frame we can write $P=\frac{1}{2} Q(1,0,0,-1)$ and $P^{\prime}=$ $\frac{1}{2} Q(1,0,0,1)$, taking the current direction as the $z$-axis. Particles in the current hemisphere $H_{c}$ have $\beta_{i}>\alpha_{i}$ while those in the proton remnant hemisphere $H_{r}$ have $\alpha_{i}>\beta_{i}$.

The momenta $p$ and $p^{\prime}$ of the initial- and final-state quarks can also be resolved along the Sudakov vectors $P$ and $P^{\prime}$. From momentum conservation,

$$
\begin{aligned}
p & =\left(1+\alpha^{\prime}+\sum \alpha_{i}\right) P \\
p^{\prime} & =\alpha^{\prime} P+\left(1-\sum \beta_{i}\right) P^{\prime}-\sum k_{t i}
\end{aligned}
$$

where

$$
\alpha^{\prime}\left(1-\sum \beta_{i}\right)=\left(\sum \vec{k}_{t i}\right)^{2} / Q^{2}
$$

The initial quark is assumed to be collinear with the proton direction and is therefore aligned along $P$. It may have an "intrinsic" transverse momentum of the order of the hadronic mass scale $\lambda \sim 1 \mathrm{GeV}$, but this will lead to corrections of order $\lambda^{2} / Q^{2}$, which we neglect. The final quark momentum $p^{\prime}$ is mostly along $P^{\prime}$; its component 
$\alpha^{\prime}$ along $P$ is small. In perturbation theory, $\alpha^{\prime}$ is of order $\alpha_{s}\left(Q^{2}\right)$ on the average, while in the soft region (all $k_{t i} \sim \lambda$ ) it is of order $\lambda^{2} / Q^{2}$. When some emissions are hard and some soft, the non-perturbative contributions to event shapes are at most of order $\alpha_{s}\left(Q^{2}\right) \lambda / Q$, which we also neglect. Thus, as far as $\lambda / Q$ corrections are concerned, $\alpha^{\prime}$ can be regarded as contributing only to the perturbative part of event shapes, and we can compute the non-perturbative part from a study of the soft region alone.

In the soft region, the matrix elements for multi-parton emission in DIS will be the same as those for $e^{+} e^{-}$annihilation into quark-antiquark plus soft partons at c.m. energy-squared $Q^{2}$, with the outgoing antiquark replaced by an incoming quark of momentum $p$. This means that we shall be able to take the results for various matrix element integrals directly from the study of $e^{+} e^{-}$event shapes [13], adjusted according to the definition of DIS event shapes.

\section{DIS event shape variables}

\subsection{Current jet thrust}

Consider first the current jet thrust $T_{Q}$, defined by the sum of longitudinal momenta in the current hemisphere normalized to $Q / 2$ :

$$
T_{Q}=\frac{2}{Q} \sum_{a \in H_{c}} p_{z a}=\frac{2}{Q}\left(p_{z}^{\prime}+\sum_{H_{c}} k_{z i}\right) .
$$

We have assumed that the outgoing quark momentum $p^{\prime}$ lies in the current hemisphere, because the probability that this is not true is of order $\alpha_{s}\left(Q^{2}\right)$, and the associated non-perturbative contribution will be at most of order $\alpha_{s}\left(Q^{2}\right) \lambda / Q$, which we neglect. In the notation of the previous section, we have

$$
\begin{aligned}
\tau_{Q} \equiv 1-T_{Q} & =\sum \beta_{i}-\sum_{H_{c}}\left(\beta_{i}-\alpha_{i}\right)+\alpha^{\prime} \\
& =\sum_{H_{r}} \beta_{i}+\sum_{H_{c}} \alpha_{i}+\alpha^{\prime}=\sum \min \left\{\alpha_{i}, \beta_{i}\right\}+\alpha^{\prime} .
\end{aligned}
$$

Where not explicitly stated, the sums extend over all partons, irrespective of which hemisphere they are in. Here the contribution of those partons that are not directly in the current hemisphere represents the loss of momentum of the outgoing quark.

Note that the expression (3.2) is additive, in the following sense: the mean value of the event shape receives a set of perturbative contributions, from $\alpha^{\prime}$ and $\min \left\{\alpha_{i}, \beta_{i}\right\}$ for hard parton emissions, plus a non-perturbative contribution from soft emissions, which can be computed from the expression

$$
\tau_{Q} \simeq \sum \min \left\{\alpha_{i}, \beta_{i}\right\}
$$


valid in the soft region, and added to the perturbative part.

If instead we measure the thrust with respect to an axis chosen to maximize its value in the current hemisphere, we obtain the same expression in the soft region, since the transverse contribution is of second order in the small quantities $k_{t i} / Q$.

Another possible way of defining the current jet thrust is to normalize it to the total energy in the current hemisphere, instead of $Q / 2$. This gives

$$
T_{E}=T_{Q} /(1-\varepsilon)
$$

where

$$
\varepsilon \equiv 1-\frac{2}{Q} \sum_{a \in H_{c}} E_{a}
$$

The quantity $\varepsilon$, which we call the energy deficit in the current hemisphere, is itself an interesting shape variable, not measured up to now. It is given by

$$
\varepsilon=\sum \beta_{i}-\sum_{H_{c}}\left(\beta_{i}+\alpha_{i}\right)-\alpha^{\prime}=\sum_{H_{r}} \beta_{i}-\sum_{H_{c}} \alpha_{i}-\alpha^{\prime}
$$

and hence in the soft region

$$
\tau_{E} \equiv 1-T_{E} \simeq 2 \sum_{H_{c}} \alpha_{i}
$$

\subsection{Current jet mass}

The current jet mass is defined by

$$
\rho=\left(\sum_{a \in H_{c}} p_{a}\right)^{2} / Q^{2}
$$

In the notation of Sect. 2 ,

$$
\rho=\left(\alpha^{\prime}+\sum_{H_{c}} \alpha_{i}\right) \cdot\left(1-\sum_{H_{r}} \beta_{i}\right)-\sum_{H_{r}} \alpha_{i} \beta_{i} .
$$

Once again we should ignore $\alpha^{\prime}$ and terms of second order in $\alpha_{i}$ and/or $\beta_{i}$ when computing $1 / Q$ corrections, in which case we have simply the additive soft contribution

$$
\rho \simeq \sum_{H_{c}} \alpha_{i}
$$

\section{$3.3 C$-parameter}

The $C$-parameter is

$$
C=6 \sum_{a, b \in H_{c}}\left|\vec{p}_{a}\right|\left|\vec{p}_{b}\right| \sin ^{2} \theta_{a b} / Q^{2}
$$


Note the factor of 6 replacing the $3 / 2$ that appears in the definition of the $C$ parameter for $e^{+} e^{-}$annihilation, because of the normalization to $(Q / 2)^{2}$ in place of $Q^{2}$.

In the notation of Sect. 2, the general expression for the $C$-parameter is complicated, but in the soft region we obtain simply

$$
C \simeq 12 \sum_{H_{c}} \frac{\alpha_{i} \beta_{i}}{\alpha_{i}+\beta_{i}},
$$

which is again additive.

\section{Soft two-loop contributions}

Consider now the contribution to a shape variable $\mathrm{V}$ when two soft, not necessarily collinear partons are emitted. Following [13], we write the two-parton phase space as

$$
d \Gamma_{2}\left(k_{1}, k_{2}\right)=\frac{d \alpha_{1}}{\alpha_{1}} \frac{d \alpha_{2}}{\alpha_{2}} \frac{d^{2} k_{t 1}}{\pi} \frac{d^{2} k_{t 2}}{\pi}=\frac{d \alpha}{\alpha} \frac{d^{2} k_{t}}{\pi} \frac{d \phi}{2 \pi} d z d m^{2}
$$

where

$$
\begin{aligned}
\alpha & =\alpha_{1}+\alpha_{2}, \quad \alpha_{1}=z \alpha, \quad \alpha_{2}=(1-z) \alpha, \\
m^{2} & =\left(k_{1}+k_{2}\right)^{2}, \quad \vec{k}_{t}=\vec{k}_{t 1}+\vec{k}_{t 2}
\end{aligned}
$$

and $\phi$ is an azimuthal angle. The contribution to the mean value of $\mathrm{V}$ from one or two soft partons, which will be denoted by $\mathcal{V}$, may be written in the form

$$
\begin{aligned}
\mathcal{V} & =4 C_{F} \int \frac{d \alpha}{\alpha} \frac{d^{2} k_{t}}{\pi k_{t}^{2}}\left(\frac{\alpha_{s}(0)}{4 \pi}+\chi\left(k_{t}^{2}\right)\right) v_{1}(k) \\
& +2 C_{F} \int d \Gamma_{2}\left(k_{1}, k_{2}\right)\left(\frac{\alpha_{s}}{4 \pi}\right)^{2}\left(C_{F} M_{F}+C_{A} M_{A}+n_{f} M_{f}\right) v_{2}\left(k_{1}, k_{2}\right),
\end{aligned}
$$

where $v_{1}(k)$ is the contribution to the shape variable from a (massless) parton of momentum $k$ and $v_{2}\left(k_{1}, k_{2}\right)$ is the corresponding two-parton contribution.

The first term on the right-hand side of Eq. (4.3) represents the lowest-order contribution of a single soft gluon (involving the ill-defined quantity $\alpha_{s}(0)$ ) plus the two-loop virtual corrections to it. The second term represents the contribution from the emission of two soft partons, $M_{F}$ etc. being the parts of the relevant matrix elements-squared with the corresponding colour/flavour factors. Both terms are divergent, but we can rewrite $\mathcal{V}$ as sum of collinear and infrared finite parts by the following procedure. We introduce a term representing a contribution from the combined parton momenta, $v_{1}\left(k_{1}+k_{2}\right)$, and split the two-parton contribution into three parts:

$v_{2}\left(k_{1}, k_{2}\right)=v_{1}\left(k_{1}+k_{2}\right)+\left[v_{1}\left(k_{1}\right)+v_{1}\left(k_{2}\right)-v_{1}\left(k_{1}+k_{2}\right)\right]+\left[v_{2}\left(k_{1}, k_{2}\right)-v_{1}\left(k_{1}\right)-v_{1}\left(k_{2}\right)\right]$. 
The first term defines the "naive massive-gluon" contribution, which treats the parton emission inclusively. The first expression in square brackets is the "non-inclusive" correction [13]. The second square bracket defines what we shall call the "nonadditive" contribution.

The combined momentum $k_{1}+k_{2}$ is massive and therefore the definition of the contribution $v_{1}\left(k_{1}+k_{2}\right)$ is somewhat ambiguous. It does not matter how we define this quantity, as long as the expression used becomes equal to $v_{1}\left(k_{1}\right)+v_{1}\left(k_{2}\right)$ in the soft and/or collinear (i.e. massless) limit. We define it as follows: if

$$
v_{1}\left(k_{i}\right)=v\left(\alpha_{i}, \beta_{i}\right), \quad \alpha_{i} \beta_{i}=k_{t i}^{2} / Q^{2}
$$

then

$$
v_{1}\left(k_{1}+k_{2}\right) \equiv v(\alpha, \beta), \quad \alpha=\alpha_{1}+\alpha_{2}, \beta=\beta_{1}+\beta_{2} ; \quad \alpha \beta=\left(k_{t}^{2}+m^{2}\right) / Q^{2} .
$$

The expression (4.3) may now be written as the sum of four finite parts, as follows [13]:

$$
\mathcal{V}=\mathcal{V}_{0}+\mathcal{V}_{i n}+\mathcal{V}_{n i}+\mathcal{V}_{n a}
$$

The first term is the so-called "naive" contribution. It incorporates only those twoloop terms proportional to the beta-function coefficient $\beta_{0}=\left(11 C_{A}-2 n_{f}\right) / 3$, which together with $\alpha_{s}(0)$ build up the running coupling, as will be explained in the following section:

$$
\mathcal{V}_{0} \equiv 4 C_{F} \int \frac{d m^{2} d k_{t}^{2}}{k_{t}^{2}+m^{2}}\left\{\frac{\alpha_{s}(0)}{4 \pi} \delta\left(m^{2}\right)-\frac{\beta_{0}}{m^{2}}\left(\frac{\alpha_{s}}{4 \pi}\right)^{2}\right\} \Omega_{0}\left(\left(k_{t}^{2}+m^{2}\right) / Q^{2}\right)
$$

where $\Omega_{0}$, the "naive trigger function", is given by

$$
\Omega_{0}(\epsilon) \equiv \int_{\epsilon}^{1} \frac{d \alpha}{\alpha} v(\alpha, \beta=\epsilon / \alpha)
$$

The second term in Eq. (4.7) is the "inclusive" correction, which combines the remaining non-Abelian part of the virtual correction with the corresponding "naive massive gluon" part of the two-parton contribution. This was shown in Ref. [13] to be given by

$$
\begin{aligned}
\mathcal{V}_{i n}= & 8 C_{F} C_{A} \int \frac{d m^{2} d k_{t}^{2}}{m^{2}\left(k_{t}^{2}+m^{2}\right)}\left(\frac{\alpha_{s}}{4 \pi}\right)^{2} \ln \left[\frac{k_{t}^{2}\left(k_{t}^{2}+m^{2}\right)}{m^{4}}\right] \\
& \cdot\left[\Omega_{0}\left(\left(k_{t}^{2}+m^{2}\right) / Q^{2}\right)-\Omega_{0}\left(k_{t}^{2} / Q^{2}\right)\right]
\end{aligned}
$$

The "non-inclusive" correction is the additive part of the remaining non-Abelian (and quark-antiquark) two-parton contribution:

$$
\mathcal{V}_{n i}=\frac{C_{F}}{\pi} \int d m^{2} d k_{t}^{2} d z d \phi\left(\frac{\alpha_{s}}{4 \pi}\right)^{2}\left(C_{A} M_{A}+n_{f} M_{f}\right) \Omega_{n i}\left(m^{2} / Q^{2}, k_{t}^{2} / Q^{2}, z, \phi\right)
$$


where the "non-inclusive trigger function" is

$$
\Omega_{n i}\left(m^{2} / Q^{2}, k_{t}^{2} / Q^{2}, z, \phi\right) \equiv \int_{\left(k_{t}^{2}+m^{2}\right) / Q^{2}}^{1} \frac{d \alpha}{\alpha}\left[v_{1}\left(k_{1}\right)+v_{1}\left(k_{2}\right)-v_{1}\left(k_{1}+k_{2}\right)\right] .
$$

Note that the Abelian $\left(C_{F}^{2}\right)$ part of this contribution is cancelled by virtual corrections [13].

Finally the "non-additive" correction is the rest of the two-parton contribution:

$\mathcal{V}_{n a}=\frac{C_{F}}{\pi} \int d m^{2} d k_{t}^{2} d z d \phi\left(\frac{\alpha_{s}}{4 \pi}\right)^{2}\left(C_{F} M_{F}+C_{A} M_{A}+n_{f} M_{f}\right) \Omega_{n a}\left(m^{2} / Q^{2}, k_{t}^{2} / Q^{2}, z, \phi\right)$

where the "non-additive trigger function" is

$$
\Omega_{n a}\left(m^{2} / Q^{2}, k_{t}^{2} / Q^{2}, z, \phi\right) \equiv \int_{\left(k_{t}^{2}+m^{2}\right) / Q^{2}}^{1} \frac{d \alpha}{\alpha}\left[v_{2}\left(k_{1}, k_{2}\right)-v_{1}\left(k_{1}\right)-v_{1}\left(k_{2}\right)\right] .
$$

All the quantities considered in Sect. 3 are additive, in the sense that $v_{2}\left(k_{1}, k_{2}\right)=$ $v_{1}\left(k_{1}\right)+v_{1}\left(k_{2}\right)$ in the soft region, and so there is no contribution $\mathcal{V}_{n a}$ in these cases.

\section{Dispersive calculation of power corrections}

Our method for estimating power corrections will assume that the QCD coupling $\alpha_{s}\left(k^{2}\right)$ can be defined down to arbitrarily low values of the scale $k^{2}$ and that it has reasonable analytic properties, i.e. no singularities other than a cut along the negative real axis. It follows that one can write the formal dispersion relation

$$
\alpha_{s}\left(k^{2}\right)=-\int_{0}^{\infty} \frac{d m^{2}}{m^{2}+k^{2}} \rho_{s}\left(m^{2}\right)
$$

where the spectral function $\rho_{s}$ represents the discontinuity across the cut,

$$
\rho_{s}\left(m^{2}\right)=\frac{1}{2 \pi i} \operatorname{Disc}\left\{\alpha_{s}\left(-m^{2}\right)\right\} \equiv \frac{1}{2 \pi i}\left\{\alpha_{s}\left(m^{2} e^{i \pi}\right)-\alpha_{s}\left(m^{2} e^{-i \pi}\right)\right\} .
$$

Our strategy will be to rewrite the results of the previous section in terms of $\rho_{s}\left(m^{2}\right)$ and then to study the effect of a non-perturbative modification to this function in the soft region. We eliminate $\alpha_{s}(0)$ from Eq. (4.8) using Eq. (5.1), and insert $\rho_{s}\left(m^{2}\right)$ in the second-order terms in place of $\alpha_{s}^{2}$ using the result

$$
\rho_{s}=-\frac{\beta_{0}}{4 \pi} \alpha_{s}^{2}-\ldots
$$

The appropriate scale for the argument of $\rho_{s}$ in this substitution is $m^{2}$, since the twoloop contribution in Eq. (4.8) generates the running coupling. We assume that the same argument can be used in Eqs. (4.10) and (4.11). Then, for example, Eq. (4.8) becomes

$$
\mathcal{V}_{0}=\int \frac{d m^{2}}{m^{2}} \rho_{s}\left(m^{2}\right) \mathcal{F}_{0}\left(m^{2} / Q^{2}\right)
$$


where the naive characteristic function $\mathcal{F}_{0}\left(m^{2} / Q^{2}\right)$ is

$$
\mathcal{F}_{0}\left(m^{2} / Q^{2}\right)=\frac{C_{F}}{\pi} \int_{0}^{Q^{2}} d k_{t}^{2}\left[\frac{\Omega_{0}\left(\left(k_{t}^{2}+m^{2}\right) / Q^{2}\right)}{k_{t}^{2}+m^{2}}-\frac{\Omega_{0}\left(k_{t}^{2} / Q^{2}\right)}{k_{t}^{2}}\right] .
$$

Similarly we may define $\mathcal{F}_{\text {in }}$ etc. for the inclusive, non-inclusive and non-additive corrections,

$$
\begin{aligned}
& \mathcal{F}_{i n}\left(m^{2} / Q^{2}\right)=-\frac{2 C_{F} C_{A}}{\pi \beta_{0}} \int \frac{d k_{t}^{2}}{k_{t}^{2}+m^{2}} \ln \left[\frac{k_{t}^{2}\left(k_{t}^{2}+m^{2}\right)}{m^{4}}\right]\left\{\Omega_{0}\left(\left(k_{t}^{2}+m^{2}\right) / Q^{2}\right)-\Omega_{0}\left(k_{t}^{2} / Q^{2}\right)\right\} \\
& \mathcal{F}_{n i}\left(m^{2} / Q^{2}\right)=-\frac{C_{F} m^{2}}{4 \pi^{2} \beta_{0}} \int d k_{t}^{2} d z d \phi\left(C_{A} M_{A}+n_{f} M_{f}\right) \Omega_{n i}\left(m^{2} / Q^{2}, k_{t}^{2} / Q^{2}, z, \phi\right) \\
& \left.\mathcal{F}_{n a}\left(m^{2} / Q^{2}\right)=-\frac{C_{F} m^{2}}{4 \pi^{2} \beta_{0}} \int d k_{t}^{2} d z d \phi\left(C_{F} M_{F}+C_{A} M_{A}+n_{f} M_{f}\right) \Omega_{n a}\left(m^{2} / Q^{2}, k_{t}^{2} / Q^{2}, z, \phi\right)\right],
\end{aligned}
$$

so that

$$
\mathcal{V}=\int \frac{d m^{2}}{m^{2}} \rho_{s}\left(m^{2}\right) \mathcal{F}\left(m^{2} / Q^{2}\right)
$$

where $\mathcal{F}$ is the two-loop corrected characteristic function,

$$
\mathcal{F}=\mathcal{F}_{0}+\mathcal{F}_{\text {in }}+\mathcal{F}_{n i}+\mathcal{F}_{n a}
$$

Non-perturbative effects at long distances are expected to give rise to a modification in the strong coupling at low scales, $\delta \alpha_{s}$, which generates a corresponding modification in the spectral function via Eq. (5.2):

$$
\delta \rho_{s}\left(m^{2}\right)=\frac{1}{2 \pi i} \operatorname{Disc}\left\{\delta \alpha_{s}\left(-m^{2}\right)\right\}
$$

Inserting this in Eq. (5.7) and rotating the integration contour separately in the two terms of the discontinuity, we obtain the following non-perturbative contribution to Eq. (5.7):

$$
\delta \mathcal{V}=\int_{0}^{\infty} \frac{d m^{2}}{m^{2}} \delta \alpha_{s}\left(m^{2}\right) \mathcal{G}\left(m^{2} / Q^{2}\right)
$$

where, setting $m^{2} / Q^{2}=\epsilon$,

$$
\mathcal{G}(\epsilon)=-\frac{1}{2 \pi i} \operatorname{Disc}\{\mathcal{F}(-\epsilon)\}
$$

Since $\delta \alpha_{s}\left(m^{2}\right)$ is limited to low values of $m^{2}$, the asymptotic behaviour of $\delta \mathcal{V}$ at large $Q^{2}$ is controlled by the behaviour of $\mathcal{F}(\epsilon)$ as $\epsilon \rightarrow 0$. We see from Eq. (5.11) that no terms analytic at $\epsilon=0$ can contribute to $\delta \mathcal{V}$. On the other hand for a square-root behaviour at small $\epsilon$,

$$
\mathcal{F} \sim a_{V} \frac{C_{F}}{2 \pi} \sqrt{\epsilon} \quad \Longrightarrow \quad \delta \mathcal{V}=-\frac{a_{V}}{\pi} \frac{\mathcal{A}_{1}}{Q}
$$


where $\mathcal{A}_{1}$ is the $q=1$ moment of the strong coupling modification $[13,15]$,

$$
\mathcal{A}_{q} \equiv \frac{C_{F}}{2 \pi} \int_{0}^{\infty} \frac{d m^{2}}{m^{2}} m^{q} \delta \alpha_{s}\left(m^{2}\right)
$$

Note that $\delta \alpha_{s}$ is a different quantity from the "effective coupling modification" $\delta \alpha_{\text {eff }}$ introduced in Ref. [16]. Their moments are related by the formula [15]

$$
A_{q} \equiv \frac{C_{F}}{2 \pi} \int_{0}^{\infty} \frac{d m^{2}}{m^{2}} m^{q} \delta \alpha_{\mathrm{eff}}\left(m^{2}\right)=\frac{\sin (\pi q / 2)}{\pi q / 2} \mathcal{A}_{q}
$$

Thus in particular $A_{1}=2 \mathcal{A}_{1} / \pi$, and all the even moments of $\delta \alpha_{\text {eff }}$ are zero.

We can express $\mathcal{A}_{1}$ in terms of the average value of $\alpha_{s}$ in the infrared region, as follows $[17,18]$. We substitute for $\delta \alpha_{s}$ in Eq. (5.13)

$$
\delta \alpha_{s}\left(m^{2}\right) \simeq \alpha_{s}\left(m^{2}\right)-\alpha_{s}^{\mathrm{PT}}\left(m^{2}\right),
$$

where $\alpha_{s}^{\mathrm{PT}}$ represents the expression for $\alpha_{s}$ corresponding to the part already included in the perturbative prediction. As discussed in Ref. [17], if the perturbative calculation is carried out to second order in the $\overline{\mathrm{MS}}$ renormalization scheme, with renormalization scale $\mu_{\mathrm{R}}^{2}$, then we have

$$
\alpha_{s}^{\mathrm{PT}}\left(m^{2}\right)=\alpha_{s}\left(\mu_{\mathrm{R}}^{2}\right)+\left[b \ln \left(\mu_{\mathrm{R}}^{2} / m^{2}\right)+k\right] \alpha_{s}^{2}\left(\mu_{\mathrm{R}}^{2}\right)
$$

where for $N_{f}$ active flavours $\left(C_{A}=3\right)$

$$
b=\frac{11 C_{A}-2 N_{f}}{12 \pi}, \quad k=\frac{\left(67-3 \pi^{2}\right) C_{A}-10 N_{f}}{36 \pi} .
$$

The constant $k$ comes from a change of scheme from $\overline{\mathrm{MS}}$ to the more physical scheme [19] in which $\alpha_{s}$ is preferably defined at low scales. Then above some infrared matching scale $\mu_{\mathrm{I}}$ we assume that $\alpha_{s}\left(m^{2}\right)$ and $\alpha_{s}^{\mathrm{PT}}\left(m^{2}\right)$ approximately coincide, so that

$$
\begin{aligned}
\mathcal{A}_{1} & \simeq \frac{C_{F}}{\pi} \int_{0}^{\mu_{\mathrm{I}}} d m\left(\alpha_{s}\left(m^{2}\right)-\alpha_{s}\left(\mu_{\mathrm{R}}^{2}\right)-\left[b \ln \left(\mu_{\mathrm{R}}^{2} / m^{2}\right)+k\right] \alpha_{s}^{2}\left(\mu_{\mathrm{R}}^{2}\right)\right) \\
& =\frac{C_{F}}{\pi} \mu_{\mathrm{I}}\left(\bar{\alpha}_{0}\left(\mu_{\mathrm{I}}\right)-\alpha_{s}\left(\mu_{\mathrm{R}}^{2}\right)-\left[b \ln \left(\mu_{\mathrm{R}}^{2} / \mu_{\mathrm{I}}^{2}\right)+k+2 b\right] \alpha_{s}^{2}\left(\mu_{\mathrm{R}}^{2}\right)\right)
\end{aligned}
$$

where

$$
\bar{\alpha}_{0}\left(\mu_{\mathrm{I}}\right) \equiv \frac{1}{\mu_{\mathrm{I}}} \int_{0}^{\mu_{\mathrm{I}}} \alpha_{s}\left(m^{2}\right) d m .
$$

Studies of event shapes in $e^{+} e^{-}$annihilation $[10,11]$ suggest that $\bar{\alpha}_{0} \simeq 0.5$ for $\mu_{\mathrm{I}}=2$ $\mathrm{GeV}$, which translates into a value of $\mathcal{A}_{1} \simeq 0.2 \mathrm{GeV}$ for $Q \sim 20-100 \mathrm{GeV}$.

\section{Power corrections to DIS event shapes}

We now use the method of the previous section to evaluate the coefficients of $1 / Q$ for the DIS shape variables defined in Sect. 3 . 


\subsection{Current jet mass}

Consider first the current jet mass, which is given in the soft region by the simplest expression, from Eq. (3.10):

$$
v(\alpha, \beta)=\alpha \Theta(\beta-\alpha)
$$

Eq. (4.9) therefore gives for the naive trigger function

$$
\Omega_{0}^{(\rho)}(\epsilon)=\sqrt{\epsilon}
$$

dropping all terms of order $\epsilon$ (i.e. order $\left.1 / Q^{2}\right)$.

In writing Eq. (6.1) we neglected the fact that the momentum fraction of the struck quark is not $x$ but $x(1+\alpha)$. If we normalize to the Born cross section at fixed $x$, this means there should be an extra factor of $q(x(1+\alpha)) / q(x)$ on the right-hand side, where $q(x)$ is the relevant quark distribution. However, by Taylor expansion we see that this factor only gives a correction of order $\epsilon$, and so we may ignore it.

Substituting Eq. (6.2) in Eq. (5.5), we obtain

$$
\mathcal{F}_{0}^{(\rho)}(\epsilon)=-2 \frac{C_{F}}{\pi} \sqrt{\epsilon} .
$$

By simple dimensional analysis, the inclusive and non-inclusive corrections to the characteristic function, given by Eqs. (5.6) and (5.6), are also proportional to $\sqrt{\epsilon}$ :

$$
\mathcal{F}_{i n}^{(\rho)}(\epsilon)=-2 \frac{C_{F}}{\pi} r_{i n} \sqrt{\epsilon}
$$

where

$$
r_{i n}=\frac{C_{A}}{\beta_{0}} \int_{0}^{\infty} \frac{2 x \ln \left[x^{2}\left(1+x^{2}\right)\right]}{\left(1+x^{2}\right)\left(x+\sqrt{1+x^{2}}\right)} d x=3.2994 \frac{C_{A}}{\beta_{0}}
$$

and

$$
\mathcal{F}_{n i}^{(\rho)}(\epsilon)=-2 \frac{C_{F}}{\pi} r_{n i} \sqrt{\epsilon}
$$

where

$$
r_{n i}=\frac{m}{8 \pi \beta_{0}} \int d k_{t}^{2} d z d \phi\left(C_{A} M_{A}+n_{f} M_{f}\right)\left(k_{t 1}+k_{t 2}-\sqrt{k_{t}^{2}+m^{2}}\right)
$$

with

$$
\begin{aligned}
& k_{t 1}^{2}=z\left[z k_{t}^{2}+(1-z) m^{2}+2 \sqrt{z(1-z)} k_{t} m \cos \phi\right] \\
& k_{t 2}^{2}=(1-z)\left[(1-z) k_{t}^{2}+z m^{2}-2 \sqrt{z(1-z)} k_{t} m \cos \phi\right] .
\end{aligned}
$$

The integration in Eq. (6.7) was performed for $e^{+} e^{-}$annihilation in Ref. [13]. As explained above, the result for DIS will be identical in the soft region, and therefore we can take the value directly from there (which we also confirmed numerically):

$$
r_{n i}=-\frac{2}{\beta_{0}}\left(0.862 C_{A}+0.052 n_{f}\right) .
$$


Since the contributions to the jet mass from multiple soft parton emission are simply additive, see Eq. (3.9), there is no non-additive correction. The two-loop corrected characteristic function for the current jet mass is thus

$$
\mathcal{F}^{(\rho)}(\epsilon)=-2 \mathcal{M} \frac{C_{F}}{\pi} \sqrt{\epsilon}
$$

where $\mathcal{M}$ is the Milan factor computed in Ref. [13],

$$
\begin{aligned}
\mathcal{M} & =1+r_{i n}+r_{n i}=1+\left(1.575 C_{A}-0.104 n_{f}\right) / \beta_{0} \\
& =1.49 \text { for } n_{f}=3 .
\end{aligned}
$$

It follows from Eqs. (5.12) and (6.10) that the leading power correction to the mean current jet mass is

$$
\delta\langle\rho\rangle=\frac{4 \mathcal{M}}{\pi} \frac{\mathcal{A}_{1}}{Q}=1.89 \frac{\mathcal{A}_{1}}{Q} \text { for } n_{f}=3
$$

Comparing with our earlier result given in Ref. [1],

$$
\delta\langle\rho\rangle \sim 2 \frac{A_{1}}{Q},
$$

we note the following differences:

- The first moment of the effective coupling modification, $A_{1}$, is replaced by $2 \mathcal{A}_{1} / \pi$ where $\mathcal{A}_{1}$ is the first moment of the modification to the strong coupling itself, in accordance with Eq. (5.14).

- The result is enhanced by a factor which is identical to the Milan factor obtained in $e^{+} e^{-}$annihilation [13].

This means that universality of $1 / Q$ corrections still holds, in the sense that the coefficient of $1 / Q$ for the DIS current jet mass remains one half of that for the thrust in $e^{+} e^{-}$annihilation, for example. Furthermore the numerical coefficient of $\mathcal{A}_{1} / Q$ is only slightly lower than the coefficient of $A_{1} / Q$ calculated earlier using the "naive massive gluon" approximation, because the Milan factor $\mathcal{M}$ is largely cancelled by the translation factor of $2 / \pi$. Thus experimental results [9] interpreted earlier as measurements of $A_{1}$ using the "naive" formula can be reinterpreted as giving a similar value for $\mathcal{A}_{1}$, taking into account the Milan and translation factors.

\subsection{Current jet thrust}

For the quantity $\tau=1-T_{Q}$ where $T_{Q}$ is the current jet thrust, we have from Eq. (3.3)

$$
v(\alpha, \beta)=\alpha \Theta(\beta-\alpha)+\beta \Theta(\alpha-\beta)
$$


and Eqs. (4.9) and (5.5) now give

$$
\Omega_{0}^{(\tau)}(\epsilon)=2 \sqrt{\epsilon}, \quad \mathcal{F}_{0}^{(\tau)}(\epsilon)=-4 \frac{C_{F}}{\pi} \sqrt{\epsilon} .
$$

Similarly, both the inclusive and non-inclusive corrections to the characteristic function are twice as large as those for the jet mass, and again there is no non-additive part, so the leading power correction is

$$
\delta\langle\tau\rangle=\frac{8 \mathcal{M}}{\pi} \frac{\mathcal{A}_{1}}{Q}=3.79 \frac{\mathcal{A}_{1}}{Q} \text { for } n_{f}=3,
$$

to be compared with our earlier result of $4 A_{1} / Q$ in Ref. [1]. Once again the two-loop result (6.16) has a similar coefficient, but $A_{1}$ is replaced by $\mathcal{A}_{1}$.

As stated earlier, the above result for the $1 / Q$ power correction (unlike the perturbative contribution) does not depend on whether the thrust is measured relative to the current axis or an axis which maximizes its value. We also see from Eq. (3.7) that the same leading power correction should be obtained if the thrust is normalized to the total energy in the current hemisphere, instead of $Q / 2$. This is because the mean energy deficit in the current hemisphere, $\varepsilon$, defined in Eq. (3.5), should have no $1 / Q$ correction, since the two terms in Eq. (3.6) cancel on the average. ${ }^{1}$

\section{$6.3 C$-parameter}

Similarly for the $C$-parameter (3.11), we have

$$
v(\alpha, \beta)=12 \frac{\alpha \beta}{\alpha+\beta} \Theta(\beta-\alpha)
$$

and the naive trigger function is

$$
\Omega_{0}^{(C)}(\epsilon)=3 \pi \sqrt{\epsilon},
$$

giving

$$
\mathcal{F}_{0}^{(C)}(\epsilon)=-6 C_{F} \sqrt{\epsilon} .
$$

The inclusive and non-inclusive corrections scale in the same way and again there is no non-additive part, so

$$
\delta\langle C\rangle=12 \mathcal{M} \frac{\mathcal{A}_{1}}{Q}=17.88 \frac{\mathcal{A}_{1}}{Q} \text { for } n_{f}=3,
$$

Our one-loop result for $C$ in Ref. [1] was ambiguous, since it depended on how the gluon mass was included. Following the definition used here, the "naive" result is $6 \pi A_{1} / Q=18.8 A_{1} / Q$. So again the two-loop result (6.20) has a similar coefficient, but with $A_{1}$ replaced by $\mathcal{A}_{1}$.

\section{Conclusions}

\footnotetext{
${ }^{1}$ We expect the leading non-perturbative correction to $\langle\varepsilon\rangle$ to be of order $\alpha_{s}\left(Q^{2}\right) / Q$.
} 
Our results on the coefficients of the leading power corrections to the DIS event shapes defined in Sect. 3 are summarized in Table 1 . For all the shape variables studied, we have found that, as in $e^{+} e^{-}$annihilation [13], the enhancement factor for the leading power correction is equal to the Milan factor (6.11), provided the "naive" coefficient is computed in the

\begin{tabular}{|c|cc|}
\hline $\mathcal{V}$ & 1-loop & 2-loop \\
\hline$\langle\rho\rangle$ & 2 & 1.9 \\
$\langle\tau\rangle$ & 4 & 3.8 \\
$\langle C\rangle$ & $6 \pi-12 \pi$ & 17.9 \\
\hline
\end{tabular}

Table 1: Coefficients of $A_{1} / Q$ (1-loop) and $\mathcal{A}_{1} / Q$ (2-loop). way specified in Sect. 4. This eliminates the ambiguity in the prediction for the $C$-parameter, which was present at the 1-loop level [1] as indicated.

In all cases the enhancement factor practically cancels the suppression factor of $2 / \pi$ that comes from translating the first moment of the effective coupling modification, $A_{1}$, into the corresponding quantity for the strong coupling itself, $\mathcal{A}_{1}$. This quantity can be used to infer the value of $\bar{\alpha}_{0}$, the low-energy moment of $\alpha_{s}$ defined in Eq. (5.19).

The experimental data on thrust and jet masses in both DIS $[9]^{2}$ and $e^{+} e^{-}$ annihilation $[10,11]$ suggest a value of $\bar{\alpha}_{0} \simeq 0.5$. Final experimental results on the $C$-parameter in DIS have yet to be presented.

\section{Acknowledgments}

We are most grateful to Yu.L. Dokshitzer, A. Lucenti, G. Marchesini and G.P. Salam, and also to members of the H1 Collaboration, especially H.-U. Martyn and K. Rabbertz, for many helpful discussions and comments. M.D. acknowledges the financial support of Trinity College, Cambridge and the comments and contributions of G.E. Smye and L. Magnea, the co-authors of Ref. [20], where an independent calculation led to the discovery and rectification of an error in the numerical value of $\mathcal{M}$.

\section{References}

[1] M. Dasgupta and B.R. Webber, Eur. Phys. J. C1 (1998) 539 (hep-ph/9604297).

[2] B.R. Webber, Phys. Lett. B 339 (1994) 148.

[3] M. Beneke, V.M. Braun and V.I. Zakharov, Phys. Rev. Lett. 73 (1994) 3058;

P. Ball, M. Beneke and V.M. Braun, Nucl. Phys. B 452 (1995) 563 (hep-ph/9502300); M. Beneke and V.M. Braun, Phys. Lett. B 348 (1995) 513 (hep-ph/9411229), Nucl. Phys. B 454 (1995) 253 (hep-ph/9506452); M. Beneke, V.M. Braun and L. Magnea, Nucl. Phys. B 497 (1997) 297 (hep-ph/9701309).

\footnotetext{
${ }^{2}$ The thrust and jet mass values we refer to here are called $T_{c}$ and $\rho_{c}$ in Ref. [9]. For these quantities the coefficients used there to fit the data were close to those given in Table 1.
} 
[4] M. Neubert, Phys. Rev. D 51 (1995) 5924 (hep-ph/9412265).

[5] A.H. Mueller, Nucl. Phys. B 250 (1985) 327; in QCD 20 Years Later, vol. 1 (World Scientific, Singapore, 1993); Phys. Lett. B 308 (1993) 355.

[6] V.I. Zakharov, Nucl. Phys. B 385 (1992) 452; lectures at YKIS97 (hep-ph/9802416).

[7] G.P. Korchemsky and G. Sterman, Nucl. Phys. B 437 (1995) 415 (hep-ph/9411211); in Proc. 30th Rencontres de Moriond, Meribel les Allues, France, March 1995 (hep-ph/9505391); G.P. Korchemsky, G. Oderda and G. Sterman, in Proc. 5th Int. Workshop on Deep Inelastic Scattering and QCD (DIS 97), Chicago, April 1997 (hep-ph/9708346).

[8] R. Akhoury and V.I. Zakharov, Phys. Lett. B 357 (1995) 646 (hep-ph/9504248); Nucl. Phys. B 465 (1996) 295 (hep-ph/9507253).

[9] H1 Collaboration, C. Adloff et al., Phys. Lett. B 406 (1997) 256 (hep-ex/9706002).

[10] DELPHI Collaboration, P. Abreu et al., Z. Physik C 73 (1997) 22; D. Wicke, in Proc. QCD97, Montpellier, July 1997, Nucl. Phys. 64 (Proc. Suppl.) (1998) 27 (hep-ph/9708467).

[11] JADE Collaboration, P.A. Movilla Fernandez et al., Eur. Phys. J. C1 (1998) 461 (hep-ex/9708034); O. Biebel, Aachen preprint PITHA-97-32 (hep-ex/9708036).

[12] P. Nason and M.H. Seymour, Nucl. Phys. B 454 (1995) 291 (hep-ph/9506317).

[13] Yu.L. Dokshitzer, A. Lucenti, G. Marchesini and G.P. Salam, Nucl. Phys. B 511 (1998) 396 (hep-ph/9707532); J. High Energy Phys. 05 (1998) 003 (hep-ph/9802381).

[14] Yu.L. Dokshitzer, A. Lucenti, G. Marchesini and G.P. Salam, J. High Energy Phys. 01 (1998) 011 (hep-ph/9801324);

Yu.L. Dokshitzer, G. Marchesini and G.P. Salam, Milan preprint in preparation.

[15] M. Dasgupta, G.E. Smye and B.R. Webber, J. High Energy Phys. 04 (1998) 017 (hep-ph/9803382).

[16] Yu.L. Dokshitzer, G. Marchesini and B.R. Webber, Nucl. Phys. B 469 (1996) 93 (hep-ph/9512336).

[17] Yu.L. Dokshitzer and B.R. Webber, Phys. Lett. B 352 (1995) 451 (hep-ph/9504219).

[18] Yu.L. Dokshitzer and B.R. Webber, Phys. Lett. B 404 (1997) 321 (hep-ph/9704298).

[19] S. Catani, G. Marchesini and B.R. Webber, Nucl. Phys. B 349 (1991) 635.

[20] M. Dasgupta, L. Magnea and G.E. Smye, J. High Energy Phys. 9911 (1999) 025 